Unfallchirurg 2013 · 116:196-197

DOI 10.1007/s00113-012-2328-3

Online publiziert: 3. März 2013

(c) Springer-Verlag Berlin Heidelberg 2013

M.J.B. Keel

Universitätsklinik für Orthopädische Chirurgie und Traumatologie, Inselspital, Universitätsspital Bern

\title{
Beckenring und Acetabulum
}

der häufigsten bewährten aber auch neuen Zugänge in der Becken- und Acetabulumchirurgie dargestellt. Becker et al. der Homburger Beckengruppe von Tim Pohlemann beschreiben in ihrem Artikel die Präperationsschritte und die Indikationen anteriorer Zugänge zum Beckenring in Rückenlage, Lehmann et al. der Hamburger Arbeitsgruppe von Johannes Rüger die posterioren Zugänge in Bauchlage. Chirurgen aus Bern, Theodor Kocher am Ende des 19. Jahrhunderts und Reinhold Ganz am Ende des 2. Jahrtausends, hatten die Entwicklung der Acetabulumchirurgie maßgeblich geprägt [2]. Die aktuelle Berner Beckengruppe um Klaus Siebenrock und Marius Keel versucht deshalb die Geschichte und die Eigenheiten vorderer und hinterer Zugänge zum Acetabulum aufzuzeigen. Im letzten Artikel stellen Ossendorf et al. der Mainzer Gruppe von Pol Rommens Entscheidungsmerkmale anhand illustrativer Beispiele dar, die für die Zugangsund Lagerungswahl bei der Akut- oder Definitivversorgung von Beckenfrakturen von Bedeutung sind.
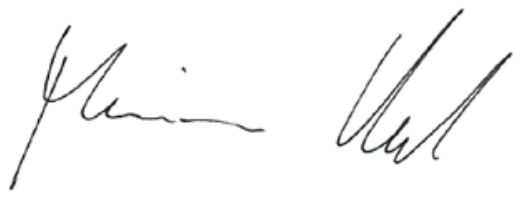

M.J.B. Keel

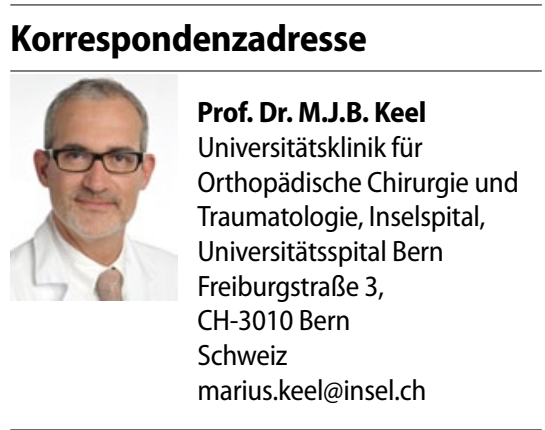

Literatur

1. Keel MJ, Ecker TM, Cullmann JL et al (2012) The Pararectus approach for anterior intrapelvic management of acetabular fractures. An anatomical study and clinical evaluation. J Bone Joint Surg Br 94:405-411

2. Keel MJ, Ecker TM, Siebenrock KA, Bastian JD (2012) Rationales for the Bernese approaches in acetabular surgery. Eur J Trauma Emerg Surg 38:489-498

3. Keel M, Trentz O (2005) Acute management of pelvic fractures. Curr Orthop 19:334-344

4. Rommens PM, Wagner D, Hofmann A (2012) Surgical management of osteoporotic pelvic fractures: a new challenge. Eur J Trauma Emerg Surg 38:499509 
Hier steht eine Anzeige.

黛 Springer 